\title{
THE HOPF'S LIMITING CYCLE
}

\section{- A METHOD TO MEASURE THE ELECTRON COOLING FORCE}

\author{
K.Y. Ng \\ Fermi National Accelerator Laboratory, ${ }^{*}$ P.O. Box 500, Batavia, IL 60510
}

(September 2002)

\begin{abstract}
The longitudinal phase space structure of protons in the presence of an electron drag force is studied. Depending on the relative difference in average proton velocity and electron velocity, the fixed point near the center of the phase space can be stable or unstable. When it is unstable, a stable Hopf's limit cycle exists. By measuring the appearance and size of the Hopf's limit cycle, the electron cooling force can be deduced.
\end{abstract}

${ }^{*}$ Operated by the Universities Research Association, Inc., under contract with the U.S. Department of Energy. 


\section{ELECTRON COOLING FORCE}

For a Gaussian distribution, the electron cooling force resembles the derivative of a Gaussian with its extrema determined by the rms velocity spread of the electron beam. However, the velocity spread is in general much smaller in the longitudinal than the transverse directions. The cooling force may exhibit two peaks instead. Understanding the cooling force is crucial in understanding the electron cooling mechanism. In this paper, we try to understand the appearance and disappearance of the Hopf's limit cycle, from which the electron cooling force can be deduced. A solvable example is given. The analytic solution is compared with simulations. Part of this paper was first written in 1995 to explain experimental results [1] gathered at the Cooler Ring of the Indiana University Cyclotron Facility (IUCF). Some additions are included later.

\section{FIXED POINT}

The equations of motion of a proton to be cooled in the longitudinal phase space with electron cooling are

$$
\begin{aligned}
& \delta_{n+1}=\delta_{n}-\frac{2 \pi \nu_{s}^{2}}{h \eta}\left(\sin \phi_{n}-\sin \phi_{s}\right)-f\left(\delta_{n}-\delta_{e}\right) \\
& \phi_{n+1}=\phi_{n}+2 \pi h \eta \delta_{n+1} .
\end{aligned}
$$

where $\eta<0$ here for the Cooler Ring at IUCF because the machine is below transition. The cooling force is $f\left(\delta-\delta_{e}\right)$ which is so defined that it is antisymmetric with respect to its argument. Here $\delta$ is the fractional momentum offset of the particle with respect to the synchronous particle which has a synchronous rf phase of $\phi_{s}$, and $\delta_{e}$ is the fractional momentum deviation of a proton traveling at the same mean velocity as the electrons. To make the two equations of motion more symmetric, let us redefine the momentum offset as

$$
\bar{\delta}=\frac{h|\eta|}{\nu_{s}} \delta
$$

Then (2.2) becomes

$$
\begin{aligned}
\bar{\delta}_{n+1} & =\bar{\delta}_{n}+2 \pi \nu_{s}\left(\sin \phi_{n}-\sin \phi_{s}\right)-\frac{h|\eta|}{\nu_{s}} f\left(\delta_{n}-\delta_{e}\right), \\
\phi_{n+1} & =\phi_{n}-2 \pi \nu_{s} \bar{\delta}_{n+1} .
\end{aligned}
$$


For $\phi_{s}=0$ and $\phi_{n} \ll 1$, we have

$$
\begin{aligned}
& \frac{d \bar{\delta}}{d n}=2 \pi \nu_{s} \phi-\frac{h|\eta|}{\nu_{s}} f\left(\delta-\delta_{e}\right) \\
& \frac{d \phi}{d n}=-2 \pi \nu_{s} \bar{\delta}
\end{aligned}
$$

Or

$$
\frac{d^{2} \bar{\delta}}{d n^{2}}+f^{\prime}\left(\delta-\delta_{e}\right) \frac{d \bar{\delta}}{d n}+\left(2 \pi \nu_{s}\right)^{2} \bar{\delta}=0
$$

where the $f^{\prime}$ is derivative with respect to $\delta$. Because of the presence of a frictional force, a Hamiltonian does not exist. Nevertheless, we can still study the fixed point using the equations of motion. The fixed point is given by

$$
\begin{aligned}
\bar{\delta}_{0} & =0, \\
\phi_{0} & =\frac{h|\eta|}{2 \pi \nu_{s}^{2}} f\left(-\delta_{e}\right) .
\end{aligned}
$$

Whether the fixed point is stable or not depends on

$$
f^{\prime}\left(-\delta_{e}\right)=\left\{\begin{array}{rr}
>0 & \text { stable } \\
<0 & \text { unstable }
\end{array}\right.
$$

When $\delta_{e}=0$, or exact matching between mean proton velocity and mean electron velocity, this fixed point is at the center of the phase space and is stable. This means that all protons will be damped towards the center of phase space. Of course, there are always random noise excitations, so that the eventual bunch is of finite size instead of one point. Because of the damping, this stable fixed point is also known as an attractor.

When $\delta_{e}<0$, or the mean electron velocity lags behind the synchronous proton, the situation is of Fig. 1(a). If the shift is not up to the peak of the cooling curve, $f^{\prime}$ at $\bar{\delta}=0$ remains positive. The fixed point is at $\bar{\delta}_{0}=0$ and $\phi_{0}=f\left(-\delta_{e}\right) h|\eta| /\left(2 \pi \nu_{s}^{2}\right)$ and is stable. Protons will be damped to this point instead of the center of phase space.

\section{HOPF'S LIMITING CYCLE}

As $\delta_{e}$ becomes more negative, situation like Fig. 1(b) occurs, where $f^{\prime}<0$ at $\bar{\delta}=0$. The fixed point $\bar{\delta}_{0}=0$ and $\phi_{0}=f\left(-\delta_{e}\right) h|\eta| /\left(2 \pi \nu_{s}^{2}\right)$ becomes unstable. This means that a particle 


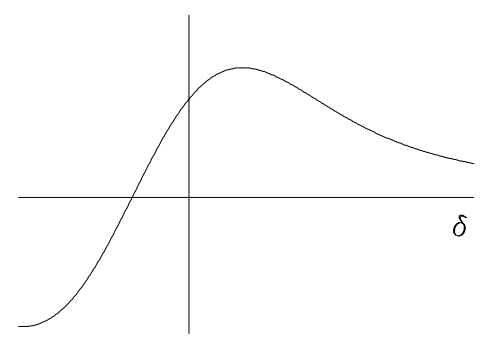

(a)

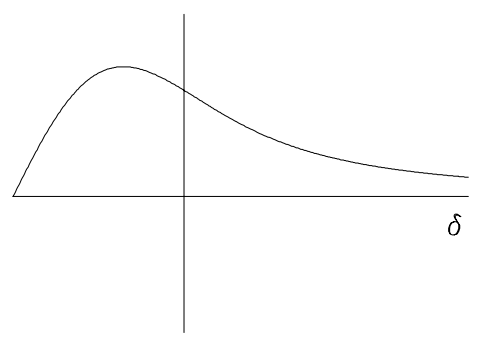

(b)

Figure 1: (a) The fixed point is stable when the electron velocity lag is not up to the peak of the cooling force. (b) The fixed point becomes unstable and bifurcates into the Hopf's limiting cycle when the electron velocity lag is big enough to pass the peak of the cooling force.

there will be repelled. It will be oscillatory about the fixed point but with larger and larger $|\bar{\delta}|$. This is because $f^{\prime}$ remains negative on both sides of $\bar{\delta}=0$. When $|\bar{\delta}|$ is big enough to pass the peak of the cooling curve, part of the oscillatory trajectory will experience $f^{\prime}>0$ and damping occurs. When the amplitude of oscillation reaches such a value that the growing is balanced by damping, the trajectory becomes stable. This is because if the amplitude is too big, the particle will experience more damping when $f^{\prime}>0$ than growing when $f^{\prime}<0$, so that the amplitude starts to reduce. This stable trajectory is unique and is called the Hopf's limit cycle. Note that this cycle is not a circle. It is obviously not symmetric about the unstable fixed point $\bar{\delta}=0$, because the cooling curve itself is not symmetric about $\bar{\delta}=0$.

Note that as is depicted in Fig. 1(b), the limiting cycle must enclose a peak of the cooling curve, so that there are regions for $f^{\prime}>0$ and $f^{\prime}<0$. Also, for the balance to occur, $f^{\prime}>0$ on the left side of the peak must be larger than $\left|f^{\prime}\right|$ in the growing region, because the trajectory excursion with $f^{\prime}<0$ is much larger than the excursion with $f^{\prime}>0$.

If the electron velocity lags behind the synchronous proton velocity more and more, the unstable fixed point will be farther and farther towards the right side of the peak of the cooling curve. The Hopf's limiting cycle will become larger and larger.

The following is the development of the longitudinal phase space $(\bar{\delta}-\phi)$, as the electron velocity starts lagging behind the synchronous proton velocity. The fixed point starts moving from the original to $\bar{\delta}=0$ and $\phi>0$. At first, the fixed point is stable. As the lagging 


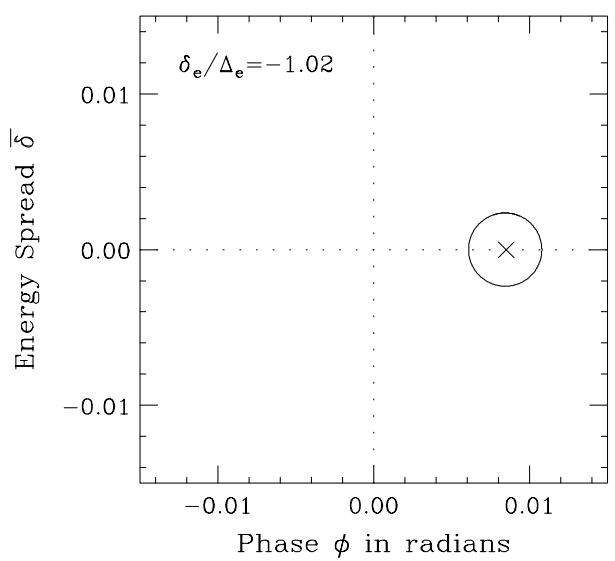

(a)

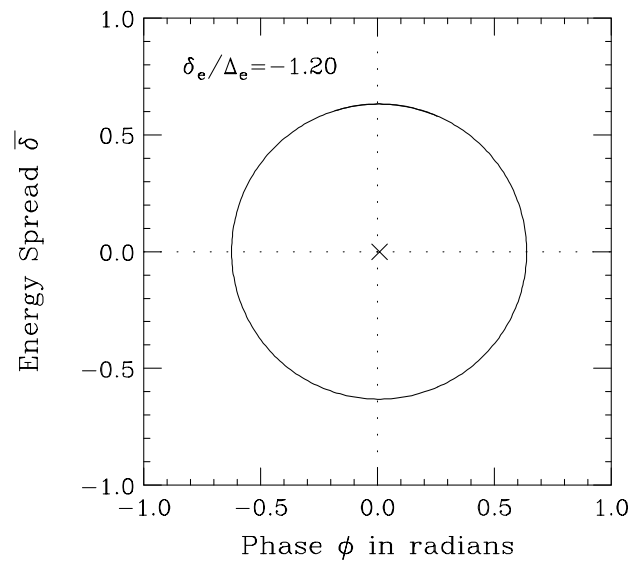

(b)

Figure 2: (a) At the start of the Hopf's bifurcation, the limiting cycle is small. (b) As the velocity lag of the electrons increases, the limiting cycle increases in size rapidly. Note the different scales used in the two plots. The unstable fixed point is denoted by a cross.

increases so that $f\left(-\delta_{e}\right)$ reaches its peak, the fixed point gradually moves to more positive $\phi$ and suddenly becomes unstable. The stable trajectory becomes a limiting cycle. The limiting cycle starts off very small around the unstable fixed point. Gradually its size increases (as the electron velocity continues to lag more and more) and crosses the origin of phase space as depicted in Fig. 2.

We would like to give a numerical illustration. First let us define a normalized cooling force $g(\zeta)$ :

$$
f(\delta)=\frac{4 \pi \alpha \Delta_{e}}{\omega_{0}} g(\zeta),
$$

where $\alpha$ is the $e$-folding cooling rate of the bunch length or momentum spread for small relative velocities between the protons and electrons, $\Delta_{e}=\sigma_{e} /(\beta c)$ is the rms spread of the electron longitudinal velocity relative to the velocity of the synchronous proton, and $\zeta=\left(\delta-\delta_{e}\right) / \Delta_{e}$. Defined in this way,

$$
g(\zeta) \rightarrow \zeta \quad \text { as } \zeta \rightarrow 0
$$

We further use

$$
g(\zeta)=\zeta e^{-|\zeta|},
$$

which peaks at $\zeta= \pm 1$. Although this form of $g(\zeta)$ does not have the correct $\zeta^{-2}$ behavior when $\zeta \rightarrow \pm \infty$, this function is simple and will well serve the purpose of the illustration. The 
parameters for a situation of an IUCF experiment will be used. This includes revolution frequency $f_{0}=\omega_{0} /(2 \pi)=1.03168 \mathrm{MHz}$, rf harmonic $h=1$, synchrotron tune $\nu_{s}=0.0003723$, slippage factor $\eta=-0.86$, damping rate $\alpha=40 \mathrm{~s}^{-1}$, and relative electron velocity spread $\Delta_{e}=0.000300$.

For a velocity lag represented by $\zeta_{e}=\delta_{e} / \Delta_{e}$, the fixed point is at $\bar{\delta}_{0}=0$ and

$$
\phi_{0}=\frac{|\eta| h \alpha \Delta_{e}}{\pi \nu_{s}^{2} f_{0}} g(\zeta) \approx 0.0230 g(\zeta)
$$

which is very small. Its maximum is only $1.32^{\circ}$ and is difficult to measure. Below $\zeta=1$, the fixed point is stable as illustrated in Fig. 3. Above $\zeta=1$, the fixed point becomes unstable and bifurcates into the Hopf's limiting cycle, which has a rf phase spread of the order of radians and can be measured easily.

It appears that no matter how large the lag in electron velocity is, the limiting cycle always exists. However, Fig. 3 is generated from a linear rf force. In reality, the rf force is nonlinear so that separatrices exist. Therefore eventually the limiting cycle will reach the separatrices of the longitudinal bucket and all the region inside the bucket becomes unstable.

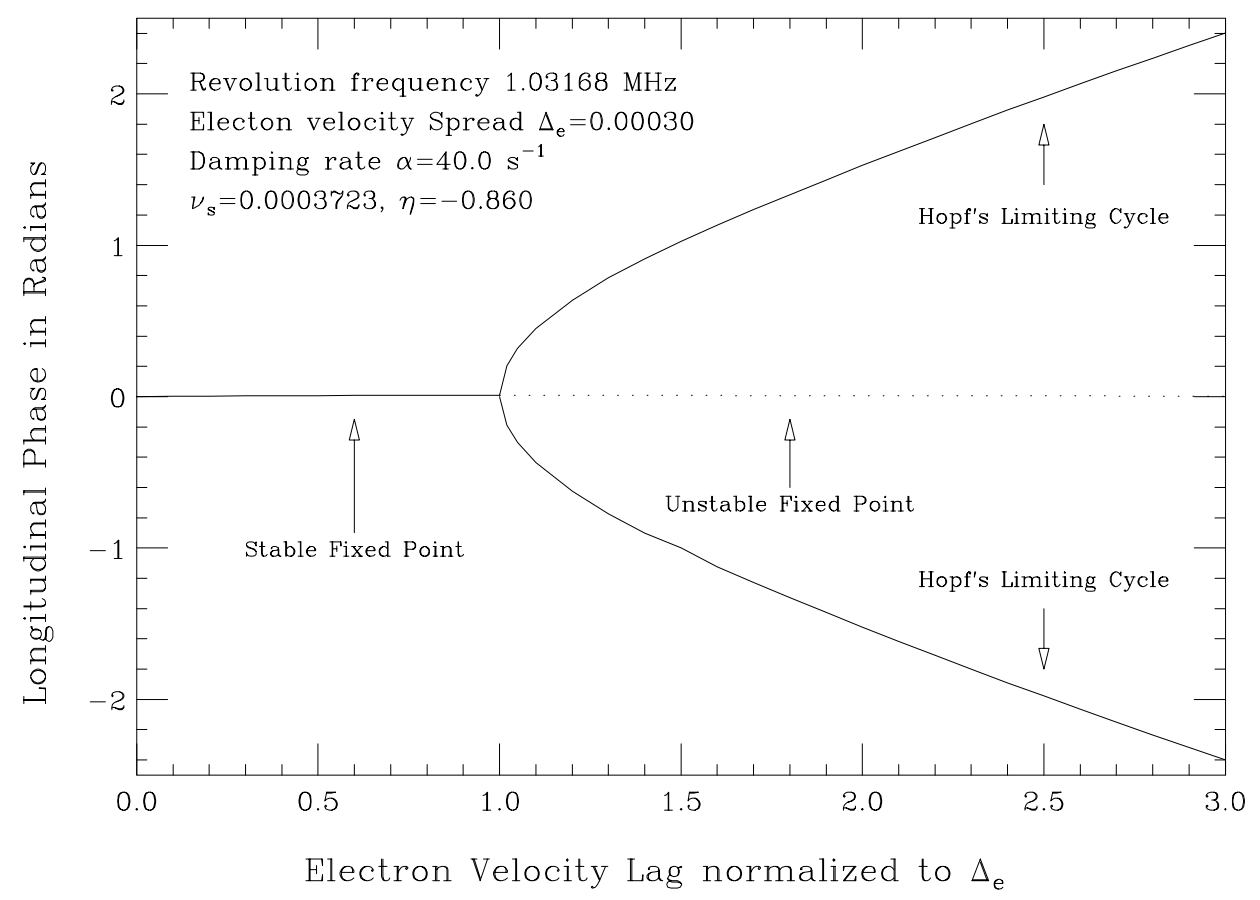

Figure 3: As the velocity lag of the electrons increases, the stable fixed point bifurcates into the Hopf's limiting cycle. 


\section{COOLING FORCE WITH DOUBLE PEAKS}

The longitudinal velocity spread $\Delta_{e \|}$ of the electrons is always much less than its transverse velocity spread $\Delta_{e \perp}$. Therefore, the cooling force may peak locally at both $\delta_{e} \approx \Delta_{e \|}$ and $\Delta_{e \perp}$, thus exhibiting two peaks and a local minimum in between. We expect the longitudinal phase space will exhibit peculiar structure in the fixed points and limiting cycles, when the velocity lag of the electron becomes as large as $\Delta_{e \|}$ and $\Delta_{e \perp}$.

A simple model to study is when the normalized cooling curve takes on the form

$$
g(\zeta)= \begin{cases}\zeta & 0 \leq \zeta<\zeta_{1} \\ \zeta_{1}+S_{1}\left(\zeta-\zeta_{1}\right) & \zeta_{1} \leq \zeta<\zeta_{2} \\ \zeta_{1}+S_{1}\left(\zeta_{2}-\zeta_{1}\right)+S_{2}\left(\zeta-\zeta_{2}\right) & \zeta_{2} \leq \zeta<\zeta_{3} \\ \zeta_{1}+S_{1}\left(\zeta_{2}-\zeta_{1}\right)+S_{2}\left(\zeta_{3}-\zeta_{2}\right)+S_{3}\left(\zeta-\zeta_{3}\right) & \zeta_{3} \leq \zeta<\zeta_{4} \\ {\left[\zeta_{1}+S_{1}\left(\zeta_{2}-\zeta_{1}\right)+S_{2}\left(\zeta_{3}-\zeta_{2}\right)+S_{3}\left(\zeta_{4}-\zeta_{3}\right)\right] \zeta_{4}^{2} / \zeta^{2}} & \zeta_{4} \leq \zeta\end{cases}
$$

and is illustrated in Fig. 4 for the parameters $\zeta_{1}=1, \zeta_{2}=2, \zeta_{3}=4, \zeta_{4}=5, S_{1}=1$,

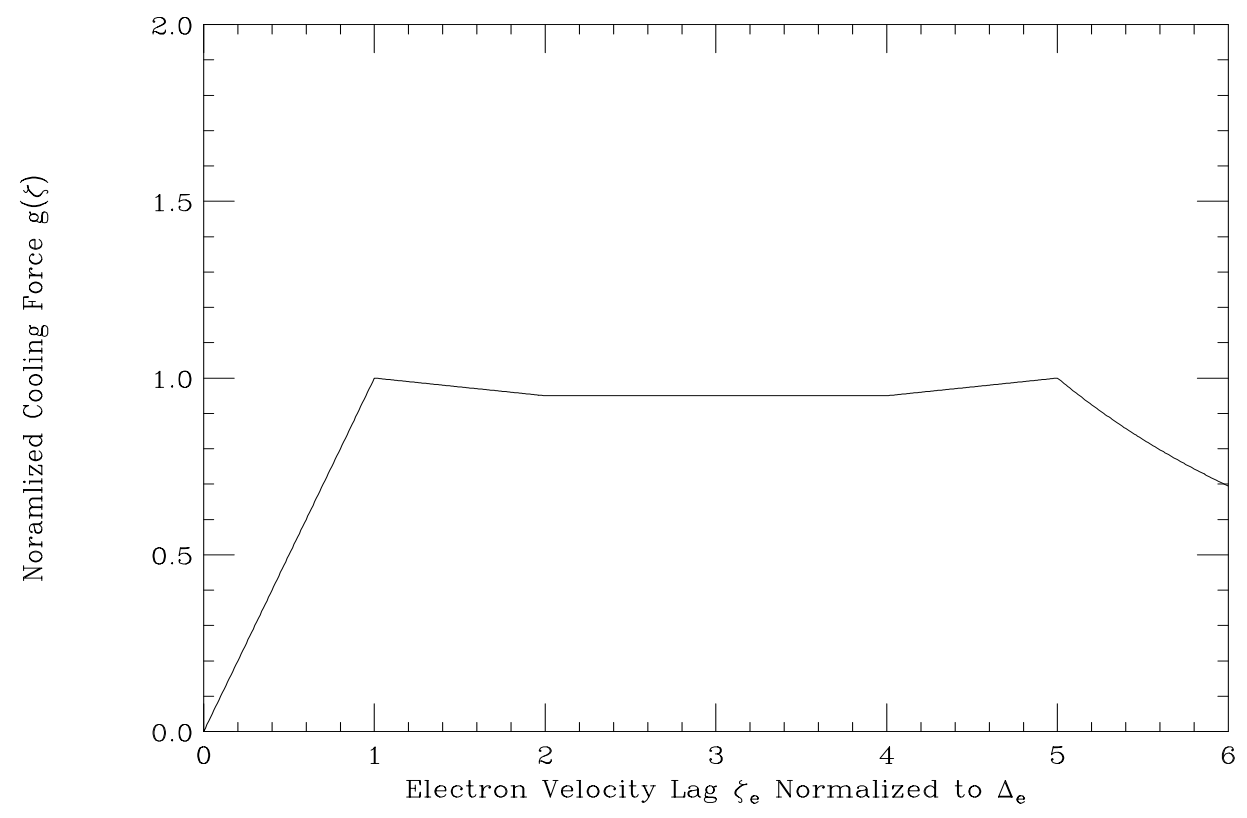

Figure 5:

The simplified normalized cooling force model with two peaks as a function of electron velocity lag.

$S_{2}=-0.05, S_{3}=0$, and $S_{4}=0.05$. Although crude and simple, this model has an 


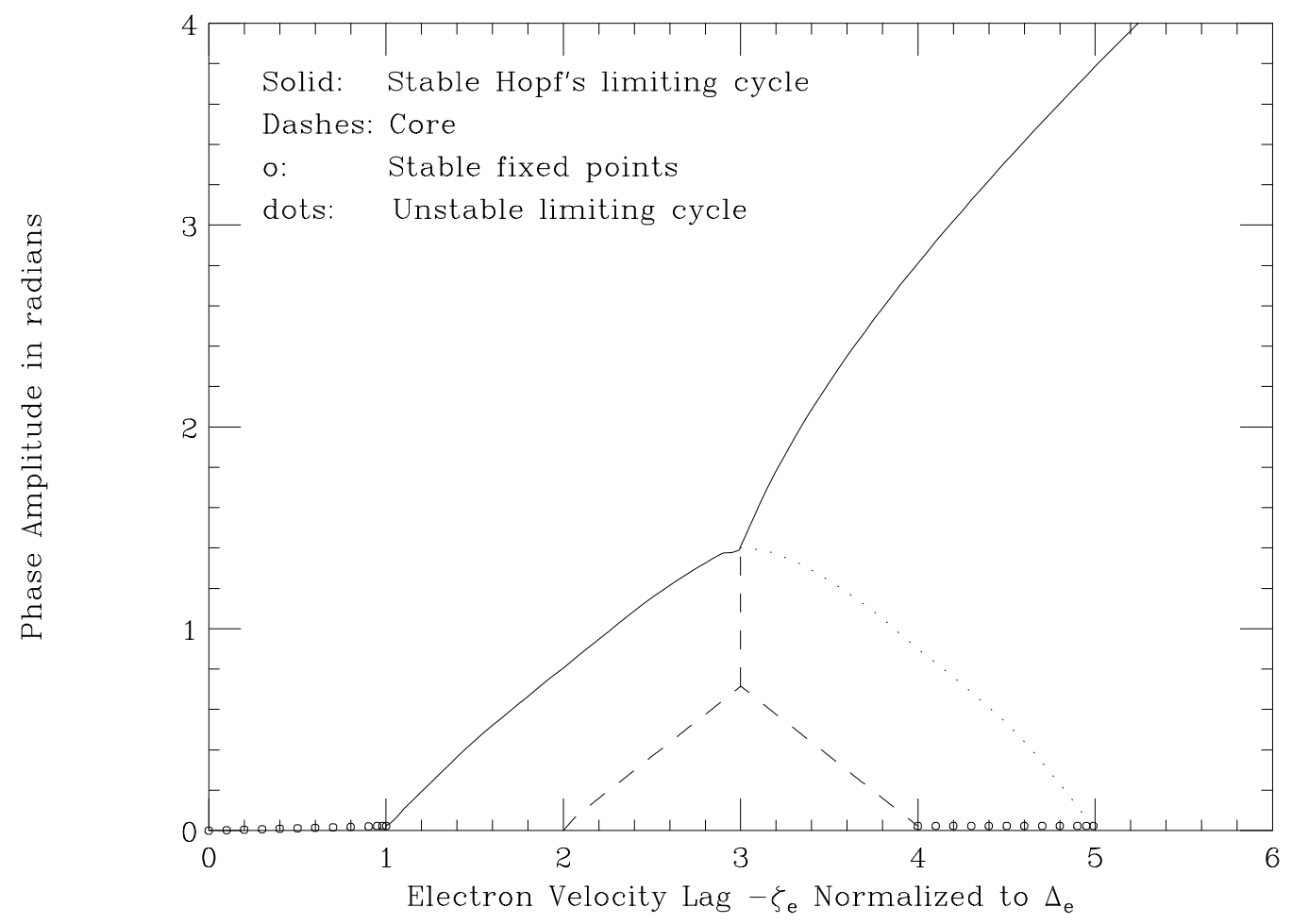

Figure 6: The simplified normalized cooling force model with two peaks as a function of electron velocity lag.

approximate analytic solution, and can therefore provide us with a clear understanding in the more general situation.

At various electron velocity lags $-\zeta_{e}$, a particle is launched from a point in the longitudinal phase space. It is tracked for many turns until it is damped toward an attractor or a stable limiting cycle. A linear rf force is first assumed, and the results are plotted in Fig. 6.

The results can also be obtained analytically and are divided into the following categories:

(1) Stable fixed point when $-\zeta_{e}<1$. When the velocity lag of the electrons is $-\zeta_{e}<1$, the fixed point is stable, because $g^{\prime}\left(-\zeta_{e}\right)>0$. The fixed point is at $\bar{\delta}_{0}=0$ and

$$
\phi_{0}=\frac{|\eta| h \alpha \Delta_{e}}{\pi \nu_{s}^{2} f_{0}} g\left(-\zeta_{e}\right)=-\frac{|\eta| h \alpha \Delta_{e}}{\pi \nu_{s}^{2} f_{0}} \zeta_{e}=-0.0230 \zeta_{e}
$$

which agrees very well with Fig. 5 .

(2) Unstable fixed point when $1<-\zeta_{e} \leq 2$. Equation (4.2) can be extended to electron 
velocity lag $1<-\zeta_{e} \leq 2$. The fixed point is at $\bar{\delta}_{0}=0$ and

$$
\phi_{0}=\frac{\eta|h| \alpha \Delta_{e}}{\pi \nu_{s}^{2} f_{0}} g\left(-\zeta_{e}\right)=\frac{\eta|h| \alpha \Delta_{e}}{\pi \nu_{s}^{2} f_{0}}\left[1-0.05\left(-\zeta_{e}-1\right)\right]=-0.0242+0.00115\left(-\zeta_{e}\right),
$$

which is unstable because $g^{\prime}<0$ in this region, and therefore cannot be obtained by tracking. (3) Stable limiting cycle when $1<-\zeta_{e}<3$. The beam particle is expelled from the unstable fixed point and performs synchrotron oscillations with larger and larger amplitudes. When the amplitude is so big that the trajectory extends into the $\zeta-\zeta_{e}<1$ region, where $\zeta=$ $\delta / \Delta_{e}$, there will be damping to counteract the growth. However, $\left|g^{\prime}\left(\zeta-\zeta_{e}\right)\right|=0.05$ when $1<\zeta-\zeta_{e}<2$, which is only $5 \%$ of the damping rate in the $\zeta-\zeta_{e}<1$ region. So the limiting cycle with balanced growth and damping can be reached when it extends only by a very small amount into this damping region. According to this logic, the limiting cycle extends approximately between $\zeta=\delta / \Delta_{e}= \pm\left(\left|\zeta_{e}\right|-1\right)$. The phase of the limiting cycle will be approximately bounded by

$$
\phi= \pm \frac{h|\eta| \Delta_{e}}{\nu_{s}}\left(\left|\zeta_{e}\right|-1\right)+\phi_{0}
$$

where the phase at the unstable fixed point is given by Eq. (4.3) for $1<-\zeta_{e} \leq 2$ and a similar expression for $2<-\zeta_{e}<3$. In above, we have made use of the fact that $\phi \sim \bar{\delta}$, according to the linearized equation of motion in Eq. (2.7). These bounds are straight lines having slopes of $\pm h|\eta| \Delta_{e} / \nu_{s}=0.693$ and reaching \pm 1.39 when $-\zeta_{e}=3$, which agree very well with the tracking results of Fig. 5. Note that this limiting cycle is stable. This is because if we enlarge the cycle by having more momentum excursion, the particle will see more damping than growth, and the trajectory will be damped back to the limiting cycle. This is because the damping slope of the cooling force is much larger than the growing slope. On the other hand, if the limiting cycle is reduced in size, there will be larger reduction in damping than growth, so that the trajectory will grow back to the limiting cycle.

(4) Stable core region at $2<-\zeta_{e}<4$. When the motion of the particle is limited to seeing only the flat portion of the cooling curve, there is no growth or damping at all. The particle is just performing synchrotron oscillations according to

$$
\begin{aligned}
& \frac{d \bar{\delta}}{d n}=2 \pi \nu_{s} \phi-\frac{2 h|\eta| \alpha \Delta_{e}}{\nu_{s} f_{0}} g\left(\zeta-\zeta_{e}\right), \\
& \frac{d \phi}{d n}=-2 \pi \nu_{s} \bar{\delta} .
\end{aligned}
$$

The particle now just sees a constant drag force because $g=0.95$ is a constant when $2<-\zeta_{e}<4$. This constant drag force amounts to a shift of the center of the synchrotron 
trajectory from the center of phase space to $\bar{\delta}=0$ and

$$
\phi_{0}=\frac{h|\eta| \alpha \Delta_{e}}{\pi \nu_{s}^{2} f_{0}} g=0.02182
$$

For example, when $-\zeta_{e}=2.5$, the particle will be free of growth and damping when the synchrotron trajectory is between $\delta / \Delta_{e}= \pm 0.5$. The phase will therefore be between $\phi= \pm\left(h|\eta| \Delta_{e} / \nu_{s}\right) 0.5+\phi_{0}=-0.325$ and 0.368 . In general, we have

$$
\phi= \begin{cases} \pm \frac{h|\eta| \Delta_{e}}{\nu_{s}}\left(\left|\zeta_{e}\right|-2\right)+\phi_{0} & 2<-\zeta_{e}<3 \\ \pm \frac{h|\eta| \Delta_{e}}{\nu_{s}}\left(4-\left|\zeta_{e}\right|\right)+\phi_{0} & 3<-\zeta_{e}<4\end{cases}
$$

where $\phi_{0}$ is given by Eq. (4.6). The positive branch hits $\phi=0.715$ at $-\zeta_{e}=3$ agreeing very well with the dashed curve in Fig. 5. Inside the triangular core region, a particle will be completely stable. Right at $-\zeta_{e}=3$, a particle will be seeing exactly the same growing force and damping force, if the synchrotron trajectory has momentum spread less than $\delta / \Delta_{e}= \pm 2$ and the trajectory will therefore be exactly stable. This corresponds to a phase spread of

$$
\phi \pm \frac{3 h|\eta| \Delta_{e}}{\nu_{s}}+\phi_{0}=-1.36 \text { or } 1.41
$$

which explains the vertical dashed line right at $-\zeta_{e}=3$.

(5) Unstable limiting cycle at $3<-\zeta_{e}<5$. A trajectory that includes the region $4<-\zeta_{e}<5$ of the cooling curve will be damped because $g^{\prime}>0$. If the trajectory extends into the $-\zeta_{e}>5$ region, there will be growth to counteract the damping so as to create a limiting cycle. Since $g^{\prime}$ starts at -0.4 at $-\zeta_{e}=5$, the growth rate will be very much larger than the damping rate $g^{\prime}=0.05$ at $4<-\zeta_{e}<5$. Thus, only a tiny excursion into this $-\zeta_{e}=5$ region will be enough to turn the damped synchrotron trajectory into a balanced limiting cycle. The half spread in momentum for this limiting cycle will be $\approx 5-\left(-\zeta_{e}\right)$, and the phase will be

$$
\phi= \pm \frac{h|\eta| \Delta_{e}}{\nu_{s}}\left(5-\left|\zeta_{e}\right|\right) .
$$

This is just the mirror image of Eq. (4.4) about $1<-\zeta_{e}<3$, and is represented by the dotted curve in Fig. 5. However, there is a big difference from Eq. (4.4) that this limiting cycle is unstable. This is due to the fact that $g^{\prime}$ for $-\zeta_{e}>5$ is bigger than $g^{\prime}$ for $-\zeta_{e}<5$. For example, if the limiting cycle is made smaller, a lot of growth from $-\zeta_{e}>5$ will disappear, so that the synchrotron trajectory will be damped either into the core if $3<-\zeta_{e}<4$ or towards a stable fixed point near the phase-space origin if $4<-\zeta_{e}<5$. The position of the 
stable fixed point is given by $\bar{\delta}=0$ and

$$
\phi=\frac{\eta|h| \alpha \Delta_{e}}{\pi \nu_{s}^{2} f_{0}} g\left(-\zeta_{e}\right)=0.0230\left(0.75-0.05 \zeta_{e}\right)
$$

to be compared with Eq. (4.2). On the other hand, if this limiting cycle is enlarged, more growth will be picked up from the $-\zeta_{e}>5$ region, and the synchrotron trajectory will increase in amplitude until it reaches the stable limiting cycle that we are going to discuss next.

(6) Stable limiting cycle at $-\zeta_{e}>3$. We just showed that the damping rate in the region $4<-\zeta_{e}<5$ region is not big enough to create a stable limiting cycle with the growth region $-\zeta_{e}>3$. In order to create such a stable limiting cycle, the particle trajectory must extend into the $-1<-\zeta_{e}<1$ region where $g^{\prime}$ or the damping rate is big. This implies that the momentum spread of this cycle must be at least from $-\zeta_{e}=1$ to $-\zeta_{e}=5$, or the phase starts between

$$
\phi= \pm \frac{2 h|\eta| \Delta_{e}}{\nu_{s}}= \pm 1.39
$$

This implies that velocity lag of the electrons must be at least $-\zeta_{e}>3$. For larger $-\zeta_{e}$, the phase of the stable limiting cycle will be larger.

When $-\zeta_{e}>5$, the cooling force should decay according to a Gaussian. Here, in our model, the cooling force decays quadratically. Thus the growth experienced by a proton in this region should be much less than the damping in the linear region when $-\zeta<1$. In other words, the effective force on a particle having excursion both in the $-\zeta<1$ region and the $-\zeta>5$ region will experience an effective damping force instead. This explains the stable Hopf's limiting cycle when $-\zeta>3$. It appears that this stable limiting cycle always exists no matter how large the electron velocity lag is. In reality, this is not true, because the rf focusing force is not linear. When the sinusoidal behavior of the rf force is taken into account, the phase is bounded by $\pi$ radians, and the corresponding tracking results are shown in Fig. 6.

\section{References}

[1] S.Y. Lee, M. Ball, B. Brabson, J. Budnick, D.D. Caussyn, P. Colestock, G. East, M. Ellison, B. Hamilton, K. Hedblom, X. Kang, D. Li, K.Y. Ng, A.Pei, A.Riabko, M. Syphers, and L. Wang, Phys. Rev. 53, 1287 (1996). 


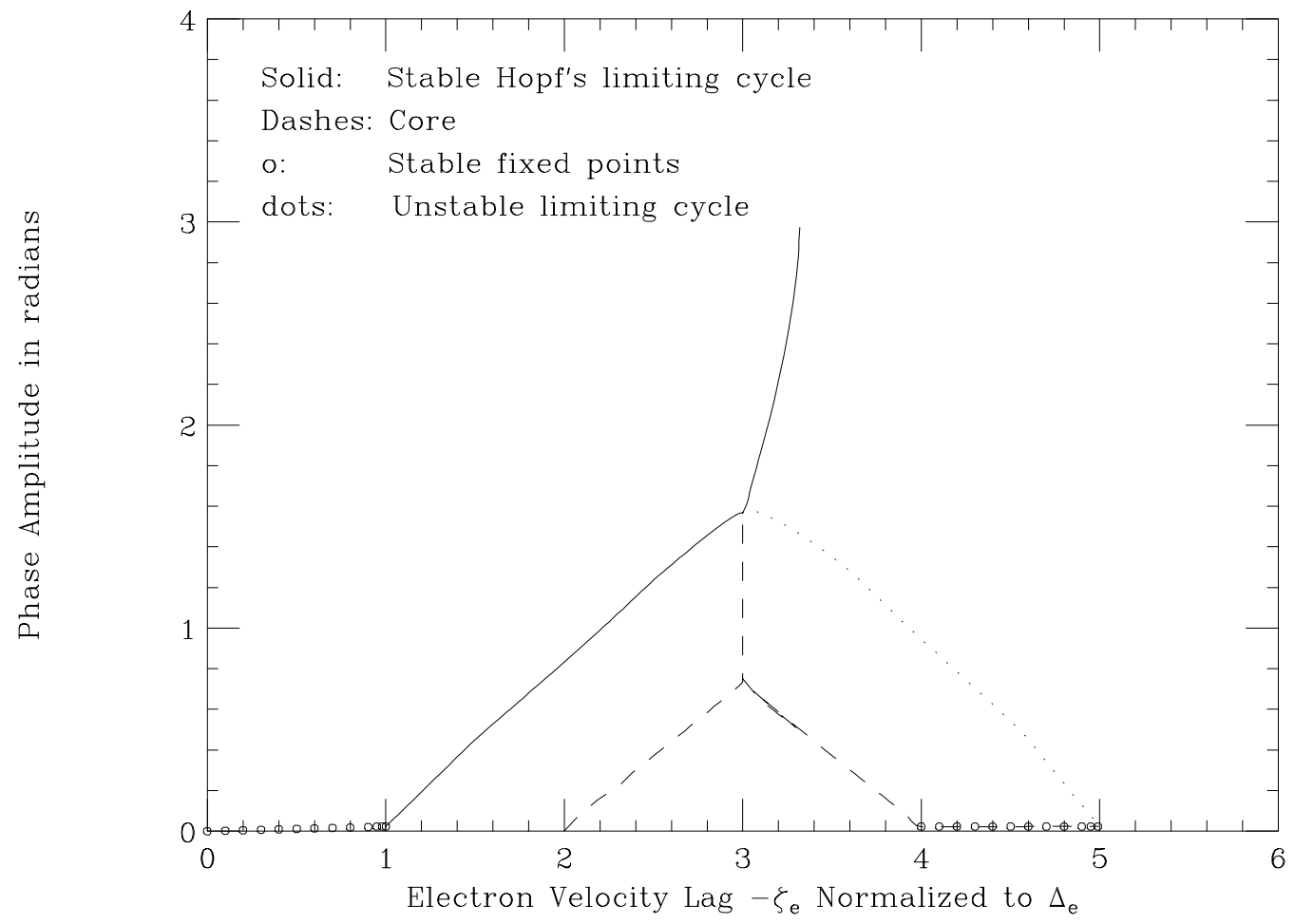

Figure 7: The simplified normalized cooling force model with two peaks as a function of electron velocity lag. 